This is a postprint version of the following published document:

González, Sergio, et al. 5G-Crosshaul: an SDN/NFV control and data plane architecture for the $5 \mathrm{G}$ integrated Fronthaul/Backhaul. Transactions on emerging telecommunications technologies, 27(9) special issue 5GPPP, Pp. 1196-1205, September 2016

DOI: https://doi.org/10.1002/ett.3066

(C) 2016 John Wiley \& Sons, Ltd. 


\title{
5G-Crosshaul: An SDN/NFV control and data plane architecture for the 5G integrated Fronthaul/Backhaul
}

\author{
Sergio González ${ }^{1 *}$, Antonio de la Oliva ${ }^{1}$, Xavier Costa-Pérez ${ }^{2}$, Andrea Di Giglio ${ }^{3}$, \\ Fabio Cavaliere ${ }^{4}$, Thomas Deiß ${ }^{5}, \mathrm{Xi} \mathrm{Li}^{2}$ and Alain Mourad ${ }^{6}$ \\ 1 Department of Telematics, Universidad Carlos III de Madrid, Madrid, Spain \\ 2 NEC Laboratories Europe, Heidelberg, Germany \\ 3 Telecom Italia, Italy \\ 4 Ericsson, Stockholm, Sweden \\ 5 Nokia, Ulm, Germany \\ 6 Interdigital Europe, UK
}

\section{ABSTRACT}

This paper presents the control and data plane architecture design for a 5G transport solution (5G-Crosshaul) with the aim of integrating the fronthaul and backhaul network segments in a common transport stratum. The control plane relies on the Software-defined networking/Network Functions Virtualization concept to control and orchestrate the different elements of the network (the 5G-Crosshaul control infrastructure). The data plane is based on an mixed optical/packetbased forwarding entity (the 5G-Crosshaul forwarding element) that leverages the benefits of optical passthrough with the statistical multiplexing of packet-based transmission, working on top of a common frame format for both, fronthaul, and backhaul traffic (the 5G-Crosshaul common frame). In addition to the main architecture design, this work includes the impact of providing multi-tenancy support into the architecture of the overall system, in order to share the costs of building and operating the infrastructure among different operators.

This architecture opens the 5G transport network as a service for innovative network applications on top (such as multitenancy, and resource management ), provisioning the required network and IT resources in a flexible, cost-effective, and abstract manner. The proposed design supports the concept of network slicing pushed by the industry for realizing a truly flexible, sharable, and cost-effective future 5G system. Copyright (C) 2016 John Wiley \& Sons, Ltd.

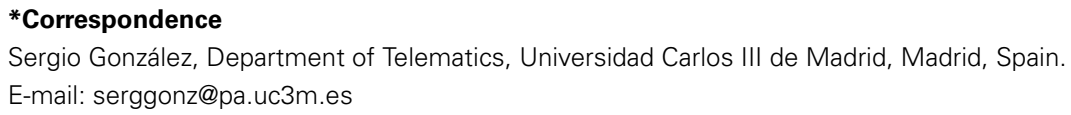

\section{INTRODUCTION}

According to the recent predictions [1] mobile data traffic will increase 8-fold between 2015 and 2020. In order to serve this increasing user demand in an environment of reduced revenues per user, a new generation of network designs is required, the so-called Fifth Generation of network architectures (5G). 5G will be characterized by an increased available bandwidth to the users, providing the user with unprecedented speeds, fostering the evolution and deployment of new services, which were not possible before. In addition, it is expected that $5 \mathrm{G}$ deployments will feature by a higher capillarity, effectively increasing the density of the network. Through this densification, spectrum can be reused in a more effective way, paving the way towards higher bandwidths available to the end user.
One key element to support the increased bandwidth to the user is the transport network that feeds the Radio Access Network. The future $5 \mathrm{G}$ transport network requires to support an unprecedented amount of traffic, with disparate requirements in terms of latency and jitter. In this context, the goal of the European H2020 5G-Crosshaul project is to build an adaptive, flexible, and softwaredefined architecture for future $5 \mathrm{G}$ transport networks integrating multi-technology fronthaul and backhaul segments. The 5G-Crosshaul architecture thus aims to enable a flexible and software-defined reconfiguration of all networking elements through a unified data plane and control plane interconnecting distributed $5 \mathrm{G}$ radio access and core network functions, hosted on in-network cloud infrastructure.

The control plane of this architecture needs to include a group of key functional elements (e.g., topology 
discovery, network monitoring, technology abstraction, and provisioning of virtual infrastructure) and their main interfaces towards the applications (northbound interface, NBI) and towards underlying technologies (southbound interface). For the design of the control plane we leverage on the software-defined network (SDN) principles and architecture defined by the Open Networking Foundation (ONF) [2] to have a unified control, management and configuration of the $5 \mathrm{G}$ multi-technology transport network and apply Network Functions Virtualization (NFV) concepts and ETSI NFV MANO architecture to enable flexible function placement and cost-effective usage of the 5G-Crosshaul infrastructure resources. The SDN principle allows the separation of the data and control planes, fostering network, and device programmability. NFV allows infrastructure and function virtualization, where the underlying physical infrastructure and network functions can be virtualized in such a way that they will be appropriately instantiated, connected, and combined over the underlying 5G-Crosshaul substrate.

The design of the data plane architecture needs to reflect the integration of heterogeneous technologies for the fronthaul and backhaul links into a single SDNbased controlled network. The main challenge of the data plane is the need for extended flexibility to adapt to the new fronthaul and backhaul technologies arising with 5G as well as to incorporate legacy technologies through abstraction interfaces.

This paper details the architecture design of the 5GCrosshaul Project towards the definition of a unified fronthaul/backhaul transport network for 5G, with special focus on the application of the multi-tenancy concept to it. The rest of the paper is structured as follows: Section 2 introduces the current motivations of the proposed unified architecture. Then, Section 3 briefly introduces the state of the art and the related work. In Section 4, we present the 5G-Crosshaul architecture, the first part, Section 4.1 is focused on an overview of the control plane (Section 4.1.1) and the data plane (Section 4.1.2) and then, the second part, Section 4.2, explains the main components of the 5G-Crosshaul architecture: (i) the 5G-Crosshaul control infrastructure (XCI) (Section 4.2.1); (ii) the 5G-Crosshaul forwarding element (XFE) (Section 4.2.2); (iii) the 5GCrosshaul Common Frame (XCF) (Section 4.2.3) and finally; and (iv) the XPU (Section 4.2.4). A preliminary analysis on the feasibility of the implementation of the proposed architecture is presented in Section 5. As explained earlier, a key feature of the 5G-Crosshaul architecture is the multi-tenancy concept with the recursive architecture of the $\mathrm{XCI}$, we summarize the different types of tenants and the layered recursive instantiation of multiple XCI over a physical infrastructure in Section 6. Finally Section 7 concludes the paper.

\section{MOTIVATION}

Current transport networks are divided into fronthaul and backhaul segments. Fronthaul links are typically point to point links used exclusively by specific fronthaul protocols, such as Common Public Radio Interface (CPRI) [3]. These links have very special properties and stringent delay and latency requirements and they are not compatible with packet-based technologies used in the rest of the network and specifically in the backhaul. For example, CPRI uses a continuous constant bitrate-like transmission, and it is not packetized. In addition, fronthaul protocols transport digitalized radio samples; hence, the bandwidth required for their transmission is proportional to the bandwidth of the radio channel and the number of antennas. This yields to the so-called bandwidth expansion, where a single LTE antenna carrier of $20 \mathrm{MHz}$ requires around $1.2 \mathrm{Gbps}$ for its transmission. This fact poses several problems to the operator that needs to dedicate expensive optical fibers for the transmission of these links and requires of different expertise for its Open Mobile Alliance operations than the rest of the network.

The bandwidth expansion suffered by digitalized radio sampling protocols rules out the applicability of these protocols to the upcoming $5 \mathrm{G}$ networks, where channel bandwidths of $100 \mathrm{MHz}$ are expected. In addition, novel techniques such as massive MIMO or even full-duplex will be used to squeeze the spectral efficiency, and using current techniques, they will require a 10 -fold increase on capacity on the 5G-Crosshaul segment, which cannot be achieved just through the evolution of current technologies. Hence, novel approaches based on different functional splits are being studied as alternatives or evolutions of current fronthaul designs. Different functional splits of the access interface impose different requirements (bandwidth, latency, jitter, and bit error rate) on the fronthaul interface. In particular, the closer the functional split to the radio frequency (RF) layer, the tighter the requirements become. That is why the requirements for the CPRI interface are quite tight, which makes this interface quite rigid (static) and costly. The move to higher functional split levels is motivated by the need to relax these transport requirements on the fronthaul interface so that lower cost and flexible means to transport the fronthaul traffic can be used. Although the network may fulfill the fronthaul requirements at a given time, it might happen that the network conditions change over time and the requirements are no longer satisfied.

In this context, a redesign of the transport network is needed, where the distinction between fronthaul and backhaul transport networks blurs as varying portions of functionality of $5 \mathrm{G}$ points of attachment (5GPoA) are moved towards the network as required for cost-efficiency reasons. The traditional capacity over-provisioning approach on the transport infrastructure will no longer be possible with 5G. Hence, a new generation of integrated fronthaul and backhaul technologies will be needed to bring the capital expenditure (CAPEX) and operational expenditure (OPEX) to a reasonable return on investment range. Also for cost reasons, the heterogeneity of transport network equipment must be tackled by unifying data, control and management planes across all technologies as much as possible. In this new integrated transport network (i.e. 
the 5G-Crosshaul), fronthaul and backhaul traffic will mix together at some forwarding nodes. The nature of the fronthaul and backhaul traffic is clearly different, and even within the fronthaul, depending on the functional split of the access interface, the traffic requirements will be different. This raises the problem of how to integrate the transport of the fronthaul and backhaul traffic in the forwarding network. The challenge here is twofold, on the data plane (transport format) and on the control plane. This is the main problem analyzed in the 5G-Crosshaul project, proposing an architecture and functional technology elements enabling a flexible integration between fronthaul and backhaul, with focus on the data and control planes.

In addition, the network operators are looking for ways to reduce CAPEX and OPEX, maximizing the use of their infrastructures. A way to optimize this objectives is by enabling a generalized and flexible sharing of the infrastructure among multiple network operators and service providers. The 5G-Crosshaul Project allows this concept through the multi-tenancy support; in a recursive and hierarchical manner, each operator (it could be virtual or not) can operate its infrastructure as the owner of the physical operates with theirs, allocating and reselling part of the resources to other operators. This concept allows a better exploit, maximizing the use of the infrastructure reducing significantly the costs.

\section{STATE OF THE ART}

The development of the new generation of mobile communication is increasing the research on new $5 \mathrm{G}$ architectures with stringent KPI requirements. Moreover, the arising of SDN and NFV as well as heterogeneous transport and access technologies is defining the $5 \mathrm{G}$ architecture design principles. These principles are being embodied in several groups and white papers (e.g., IMT-2020 (5G) Promotion Group's and NGMN's white papers [4, 5], DOCOMO's paper in [6]). In this context, it is important to highlight the work performed by the 5G-PPP Architecture Working Group White Paper ${ }^{\dagger}$ where the architecture proposed in this paper has been contributed and included.

The previously mentioned work is centered on the access or the core part of the network without considering the unification of fronthaul and backhaul segments. 5G$\mathrm{XHaul}$ project ${ }^{+}$is working on a dynamically reconfigurable optical-wireless backhaul/fronthaul with cognitive control plane for Small Cells and Cloud-RANs, differently, the 5GCrosshaul is focused on a unified control and data plane for any type of backhaul and fronthaul traffic based on SDN and NFV.

To achieve capillary network coverage, 5G-Crosshaul is designed with a mix of technologies to implement a unified backhaul and fronthaul infrastructure encompassing multiple media such as optical fibre, mmWave and

\footnotetext{
${ }^{\dagger}$ https://5g-ppp.eu/wp-content/uploads/2014/02/5G-PPP-View-on5G-Architecture-For-public-consultation.pdf.

${ }^{\dagger}$ https://5g-ppp.eu/5g-xhaul/.
}

copper wires. Among state-of-the-art systems and standards, Dense Wavelength Division Multiplexing (including NGPON2 [7]) and wireless (including mmWave [8]) are technologies that can potentially meet the $5 \mathrm{G}$-Crosshaul requirements in terms of number of users, user capacity, link distance, energy efficiency latency, cost, and scalability. However, these technologies cannot be used as they currently are but need to be adapted to the new application area. For example, Dense Wavelength Division Multiplexing needs two-order of magnitude cost reduction to be applicable to the aggregation segment in 5GCrosshaul [9], and this will only be possible by relying on new Silicon Photonics technologies for optical interfaces and switches. Together with existing, but deeply renewed, technologies, novel technologies will be considered by 5G-Crosshaul either to increase capacity and connectivity or to further reduce cost or power consumption: optical wireless technologies [10] and orthogonal frequencydivision multiplexing over fibre [11] are two examples of energy efficient solutions that can greatly enhance capacity and connectivity.

Current deployment of OBSAI [12] or CPRI [3] for the fronthaul traffic is mainly using dark fibers to the Remote Radio Heads as other technologies do not meet the stringent requirements of latency (e.g., about $100 \mu s$ for CPRI) and jitter ( $2 \mathrm{ppb}$ ). The usage of dark fibre might be acceptable for the Asian market as the deployment of existing dark fibre is very broad or the establishing of new links does not cost too much in time and money. This is not the case for other markets, especially the European market. Ethernet, due to its dominant distribution on networks and technical equipment and the initiatives to develop it further [13], is seen therefore as the base for combining fronthaul and backhaul technologies to provide cost-effective and well-accepted solutions. Because of such heterogeneity, the fronthaul and backhaul traffic of different eNBs or RRHs, even from different technologies, will share the same physical link.

The research activities of the Next Generation Fronthaul Interface Alliance (NGFI) [14], IEEE 1904.3 [13] and IEEE 802.1 [15], related to a packetized version of fronthaul traffic, are important to increase the flexibility, interoperability, and system-wide management of heterogeneous technologies of the $5 \mathrm{G}$ integrated transport network, referred here as 5G-Crosshaul.

The network programmability introduced by SDN is expected to allow a software-based control of the network through a so-called Network Operating System deployed on an external controller. In this direction, OpenDaylight ${ }^{\S}$ and ONOS $^{\text {II }}$ are the most important open source SDN controllers. ODL exposes open northbound APIs, which are used by SDN applications, the southbound interface (SBI) is capable of supporting multiple protocols, for example, OPENFLOW, BGP-LS, or PCEP. Additionally,

\footnotetext{
${ }^{\S}$ https://www.opendaylight.org/.

"http://onosproject.org/.
} 
using Network Functions Virtualization [16], virtual network functions can be run as software instances on top of an abstracted infrastructure for a more dynamic and costeffective network sharing, a few open source NFV projects are Open Source Mano", OpenStack ${ }^{* *}$, and Open Baton ${ }^{\dagger \dagger}$.

\section{5G-CROSSHAUL ARCHITECTURE}

\subsection{Overview}

The 5G-Crosshaul architecture is based on the view described in Section 1. Our design is depicted in Figure 1 and follows the traces of the SDN architecture detailed by ONF in [2] :

- Decoupled data plane and control plane;

- Control plane logically centralized;

- Exposure of state and abstract resources to applications.

\subsubsection{Control plane.}

As shown in Figure 1, the control plane is divided in two different layers: a bottom layer called 5G-Crosshaul control infrastructure (XCI) and a top layer for external applications, this top layer exploits 5G-Crosshaul resource orchestration functions to support functionalities like: planning, network and service monitoring/prediction, multi-tenancy, content delivery networks, TV Broadcasting, optimization of resources, energy management, and so on.

Successively, the XCI is our 5G Transport Management and Orchestration (MANO) platform is based on SDN/NFV principles and operates all the available resources (networking and cloud). The top layer applications can use the (NBI) offered by the XCI, typically based on REST, NETCONF, or RESTCONF APIs, to program and monitor the data plane.

The SBI, based on, for example, OPENFLOW, OFConfig, OVSDB, SNMP, and/or an ecosystem comprising several of them, will be used by the XCI to interact also with the data plane to

- Control and manage the packet forwarding behavior performed by XFEs across the 5G-Crosshaul network;

- Control and manage the PHY configuration of the different link technologies (e.g. transmission power on wireless links); and

- Control and manage the 5G-Crosshaul Processing Units (XPU) computing operations (e.g. instantiation and management of Virtual Network Functions (VNFs) via Network Function Virtualization (NFV)).

\footnotetext{
"https://osm.etsi.org/.

** https://www.openstack.org/.

${ }^{\dagger}$ http://openbaton.github.io/.
}

The scope of operation of the XCI is limited to (physical/virtual networking/storage/computing) resources within the 5G-Crosshaul transport domain.

However, the XCI will require knowledge of the configuration and or other information from the Core network and/or the Radio Access Network domains. The communication with the $5 \mathrm{G}$ Core MANO will be performed through the westbound interface and the interaction with the $5 \mathrm{G}$ Access MANO through the eastbound interface.

\subsubsection{Data plane.}

5G-Crosshaul is based on a unified, programmable, multi-tenant enable, and heterogeneous packet based transport network. This transport network is formed by XFEs (Figure 2), switching units that interconnect a broad set of link and PHY technologies under a unified frame format called 5G-Crosshaul common frame (XCF). The $\mathrm{XCF}$ is designed to handle simultaneously backhaul and fronthaul traffic, which have very different requirements, it will be possible thanks to the prioritization and timing.

The 5G-Crosshaul Processing Units (XPUs) carry out the bulk of the computing operations in the 5G-Crosshaul. The XPU shall support C-RAN, 5GPoA, functionalities virtualized (VNFs) and a heterogeneous set of other services (e.g., CDN-based services). Thereby, the NFV infrastructure (NFVI) includes all data plane (software and hardware) components that build up the networking environment in which VNFs are deployed and connected.

$\mathrm{XCI}$ can also communicate with non-5G-Crosshaul entities like BBU, legacy switches, mmWave switches, and so on using proper plugins. The 5G-Crosshaul data plane elements can communicate also with non-XCF compatible elements, thanks to a set of Adaptation Function entities (Figure 2).

\subsection{Main components}

In the following, we describe the 5G-Crosshaul main components introduced in Section 4.1

\subsubsection{G-Crosshaul control infrastructure.}

The $\mathrm{XCI}$ is the brain controlling the overall operation of the 5G-Crosshaul. The XCI follows the ETSI NFV architecture, [16]) and is formed by three main functional blocks: (i) NFV Orchestrator (NFVO), (ii) VNF Manager(s) (VNFMs), and (iii) Virtual Infrastructure Manager (VIM):

- The NFVO (NFV Orchestrator) performs the orchestration of compute, storage, and network resources available to ensure an optimized allocation and provide a network service.

- The VNFMs (VNF Managers) are responsible of the management of VNF instances (e.g., instance instantiation, modification, and termination). 


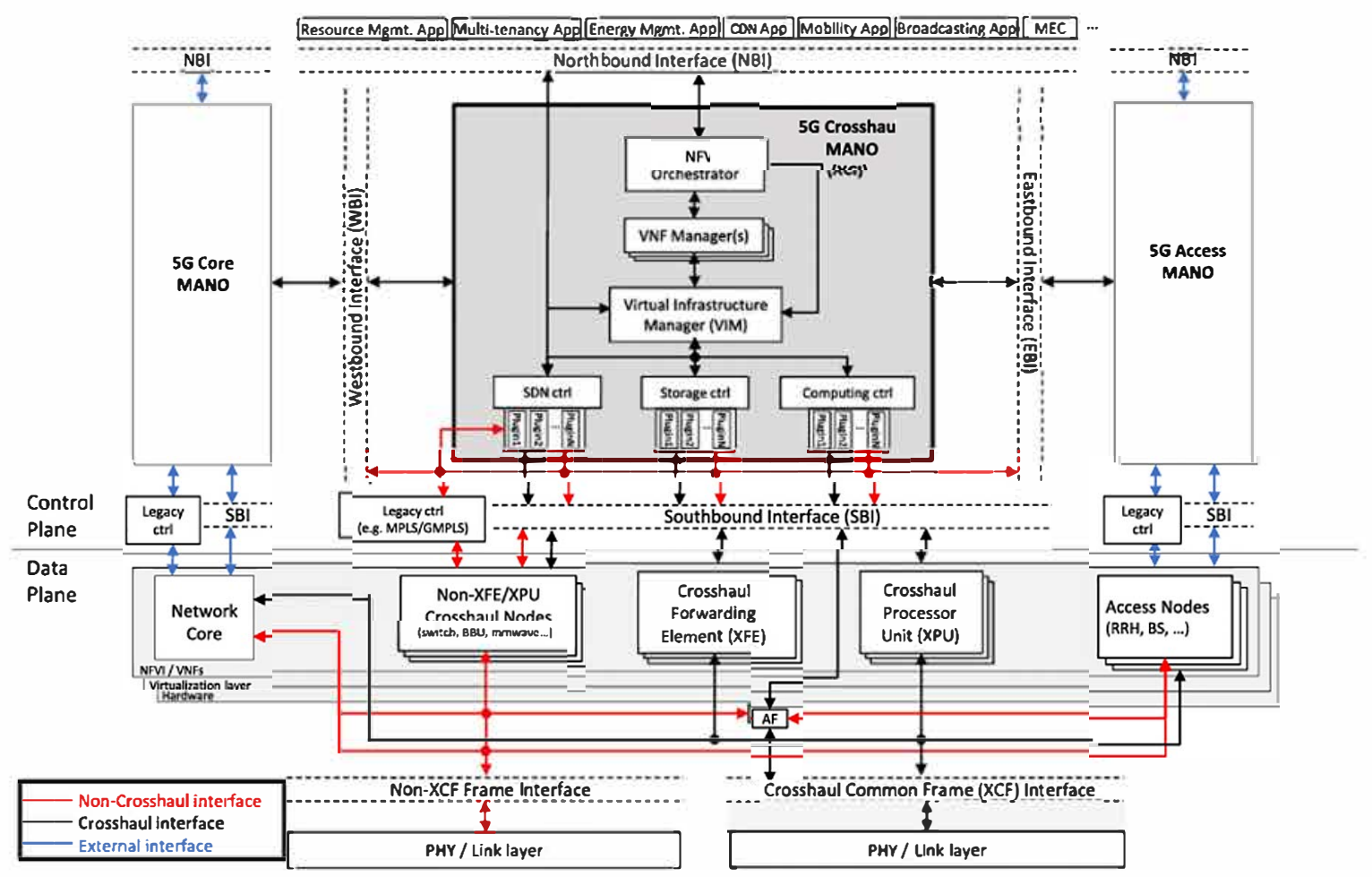

Figure 1. 5G-Crosshaul architecture.

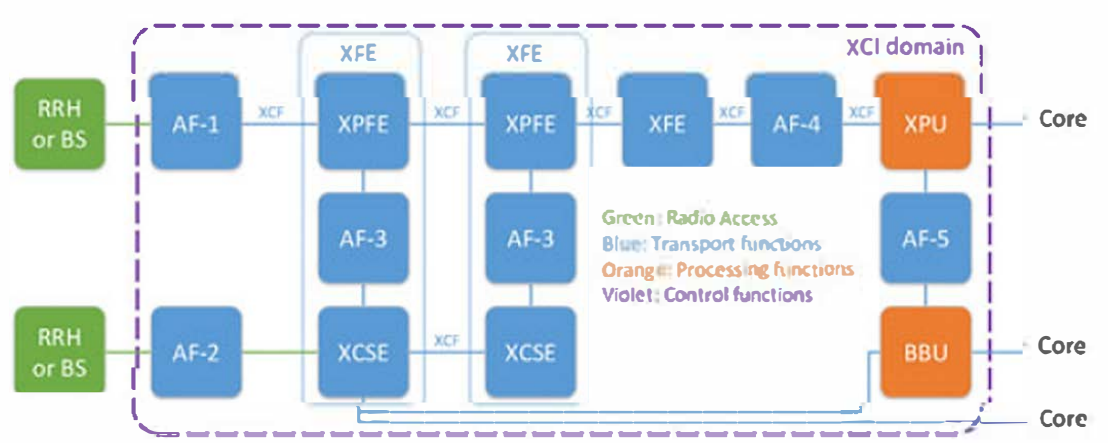

Figure 2. 5G-Crosshaul Data Plane architecture.

- The VIM (Virtualized Infrastructure Manager) controls and manages the NFVI compute, storage, and network resources.

The XCI includes a set of specialized controllers in addition to the aforementioned, their purpose is the control of the underlying network, storage, and computation resources:

- SDN Controller: is in charge of controlling the network elements following the SDN principles. 5GCrosshaul aims at extending current SDN support of multiple technologies used in transport networks (such as micro-wave links) in order to have a common SDN controlled network substrate that can be reconfigured based on the needs of the network tenants.
- Computing/Storage Controllers: Storage and Computing controllers are included in what we call a Cloud Controller. A prominent example of this kind of software framework is OPENSTACK

Note that the SDN/Computing/Storage controllers are functional blocks with one or multiple actual controllers (hierarchical or peer-to-peer structure) that centralize some or all of the control functionality of one or multiple network domains. We consider the utilization of legacy network controllers (e.g., MPLS/GMPLS) to ensure backward-compatibility for legacy equipment.

\footnotetext{
fłhttps://www.openstack.org/.
} 


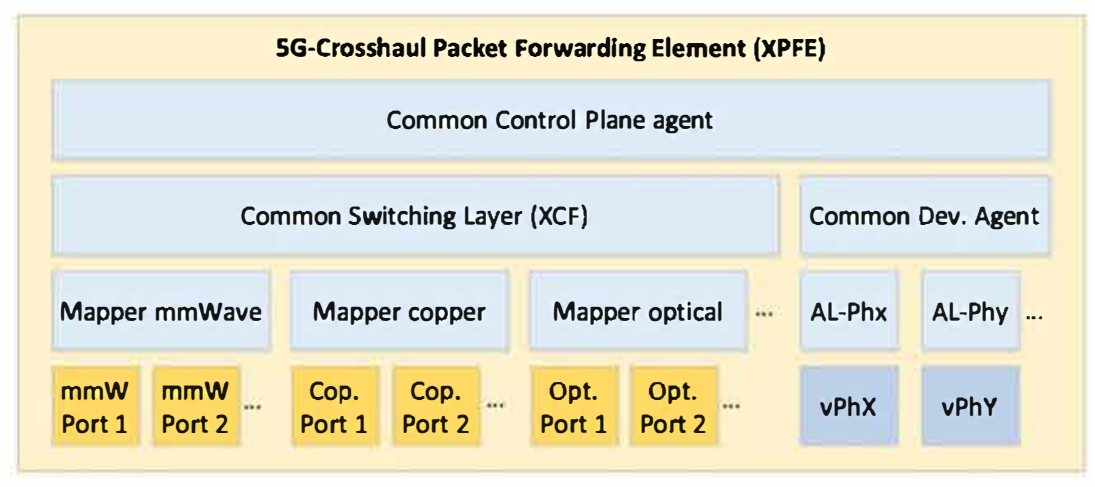

Figure 3. 5G-Crosshaul Packet Forwarding Element.

In order to ensure the feasibility of the architecture, the XCI design is based on the ETSI NFV architecture [16]. We have also considered the design and features of two well-known NFV and Cloud infrastructures: OPEN SOURCE MANO ${ }^{\S \S}$ and OPENSTACK ${ }^{\mathbf{H}}$, to guide the $\mathrm{XCI}$ design.

\subsubsection{G-Crosshaul forwarding element.}

5G-Crosshaul forwarding elements are the switching units that integrate different technologies (e.g., mmWave, Ethernet, and Optical Fibre) used in the data transmission, they enables a unified and harmonized transport traflic management. XFE supports the XCF format across the various traffic flows (of fronthaul and backhaul) and the various link technologies in the forwarding network. The XFEs are controlled by the XCI that is foreseen to have a detailed (as per the abstraction level defined) view of the fronthaul and backhaul traflic and resources, and to expose this information for intelligent resource, network functions, and topology management across the two domains.

As shown in Figure 2, XFEs are formed by packet switching elements (XPFE) and circuit switching elements (XPFE). Hence, two paths are defined: (i) a packet switching path and (ii) an optical circuit switching path. The packet switched network is the primary path for the transport of most delay-tolerant traflic, the circuit switching path complements the packet switched path for those traffic that is not suited for packet-based transport or has an extremely low delay tolerance (e.g., legacy CPRI), the circuit switching path can also be used to reduce the offload of the XPFE. This two-path switching architecture is designed to combine bandwidth efficiency, through statistical multiplexing in the packet switch, with deterministic latency ensured by the circuit switch. The modular structure of the 5G-Crosshaul switch, where layers may be added and removed, enables various deployment scenarios with traflic segregation at multiple levels, from dedicated

\footnotetext{
${ }^{88}$ https:/losm.etsi.org/.

nhttps://www.openstack.org/.
}

wavelengths to VPN, which is particularly desirable for multi-tenancy support.

Figure 3 depicts the initial architecture for the 5GCrosshaul Packet Forwarding Element (XPFE). It includes the following functions:

- A common control-plane agent to talk to the XCI.

- A common switching layer based on the XCF to forward packets between interfaces with different technologies. The switching engine is technologyagnostic and relies on (i) an abstract resource model (i.e., bandwidth, latency, bit error rate, jitter, and latency) of the underlying interfaces (i.e., mmWave, and copper), and on (ii) traffic requirements (i.e., jitter, and packet loss) that can be carried in the XCF.

- A common device agent to talk with system peripheral and expose to XCI device-related information (e.g., CPU usage, RAM occupancy, battery status and GPS position)

- Mappers for each physical interface.

- Physical interfaces with different technologies to transmit the data on the link.

The common control-plane and device agents are relevant for both packet and circuit switched forwarding elements of the XFE. It enables the creation of a technology independent and heterogeneous data-plane with dynamic reconfiguration of resources, allowing the interworking with legacy technologies.

The XFE is a critical part of the system, and as such, we have already started its prototyping. The multilayer switch design of the XFE is based on the evolution of the Ericsson Multilayer Switch, and a prototype version is already under development.

\subsubsection{G-Crosshaul common frame.}

The main goal of the 5G-Crosshaul is the integration between fronthaul and backhaul segments, the first premise needed is a common frame that can be used to transport both traffic types through the same network. XCF is the frame format used by the XPFE and is supported by all physical interfaces where packets are transported. 
The circuit switched forwarding is independent of the $\mathrm{XCF}$. Where necessary, the frame format is mapped to the XCF format for forwarding by the XPFEs (e.g., CPRI over Ethernet).

The XCF is based on Ethernet, taking advantage of MAC-in-MAC [17] (or Provider Backbone Bridged Network). MAC-in-MAC frames allow multi-tenancy, keeping the traffic of different tenants separated via the outer MAC header and allowing different forwarding behaviors per tenant. The priority of different traffic flows is enabled through the priority bits of the Ethernet header. Basing the XCF on Ethernet eases reuse of legacy switches and increases synergies with the development of more generic switches.

The use of MAC-in-MAC encapsulation as XCF ensures the feasibility of the solution, although its development requires some extensions over the OPENFLOW Protocol. Initial features in order to support MAC-in-MAC in OPENFLOW have been already included in the latest version v1.5.1 [18] of the protocol. Hence, this inclusion indicates that we are in line with the market requirements.

\subsubsection{G-Crosshaul processing unit.}

While the SDN control platform is responsible for the configuration of the network elements (i.e., the XFEs), the XPUs carry out the the bulk of the operations in the 5G-Crosshaul supporting C-RAN, 5GPoAs, VNFs, and a heterogeneous set of other services (e.g., CDN-based services). Each virtual infrastructure is instantiated, configured and operated by the XCI in the XPUs. The different functional distributions between the 5GPoA and XPU and the different services that can be hosted in the XPUs are one of the pillars of the flexibility provided by the 5G-Crosshaul architecture.

\section{IMPLEMENTATION OF THE PROPOSED SOLUTION}

This section is devoted to present a preliminary analysis performed to understand the feasibility, in terms of implementation, of the proposed architecture. First, some open source software alternatives for the XCI MANO components have been analyzed. The XCI architecture does not mandate any specific software platform; the same XCI functions can be developed starting from different open source or proprietary projects, with the unique constraint of being compliant with the interfaces defined at the NBI and SBI of each component in order to support the proper workflows and interactions. In the following, we detail the software baseline identified to be used in each functional component with their required extensions:

- NFVO: it may based on any orchestrator (OpenBaton or OSM), adding vEPC orchestration features, and (ii) a proprietary orchestrator for CDN with CDN Node orchestration

- VFNM: it may be based on (i) any orchestrator (e.g., Open Baton VNFM SDK (REST API)), adding management of vEPC VNF's lifecycle and (ii) a proprietary VNFM for CDN with orchestration of CDN nodes VNF lifecycle

- VIMaP: the VIMaP can be implemented as an extension of the VIM based on OpenStack [6] but adding the following features:

- Provisioning vEPC VNFs and their interconnections with QoS and energy constraints

- Provisioning of CDN origin and replica servers on XPUs and SFC configuration

- Allocation and management of VM and their interconnections

Storage and computing controllers will be based on state-of-the-art components, for example, based on the corresponding OpenStack modules. No further extensions have been identified for the required functionality.

As in the XCI MANO components case, the architecture does not impose any specific choice on the SDN controller software. The SDN software controllers chosen are principally OpenDayLight ${ }^{\mathrm{III}}$ and $\mathrm{ONOS}^{* * *}$. It is worth also highlighting that some of the concepts proposed in the architecture have been already implemented and contributed to the ONF Wireless Transport Proof of Concepts (PoC). The first ONF Proof of Concept PoC ${ }^{\dagger \dagger \dagger}$ on applying the SDN approach including SDN controller supporting standard open SBI and NBI for wireless transport was accomplished in October 2015, Madrid, Spain. This PoC demonstrated new optimization applications for wireless transport segments in an SDN realm, leveraging OPENFLOW extensions for management and control of wireless transport network elements. The PoC deployment includes an open sourced platform ONOS using OPENFLOW 1.3 or OPENFLOW 1.4 as the SBI, an infrastructure layer consisted of multiple types of network elements including Ethernet switches and wireless transport devices, and an application Layer consisted of defined SDN applications. The setup also includes a number of wireless transport links connected to switching aggregation nodes emulating a real deployment. Two use cases are tested in this PoC: capacity-driven air interface and flow-based shaping. The first test case shows how the SDN controller optimizes the total power consumption in a wireless transport network, while the second one is focused on dynamically adapt high throughput flows to the transport capacity currently available in the relevant section of the backhaul network. The two use-cases were tested successfully between the SDN controller and the different wireless transport and switching equipment.

The 2 nd PoC is an evolution of the first one, conducted in April 2015, Munich, Germany. This PoC focused on demonstrating the capabilities and benefits of utilizing a common microwave information model for multi-vendor

\footnotetext{
IIII https://www.opendaylight.org/.

**** http://onosproject.org/.

${ }^{\dagger \dagger} \mathrm{http} / / / 5 \mathrm{~g}$-crosshaul.eu/wireless-transport-sdn-proof-of-concept/.
} 
control of wireless network elements through open management interfaces. The PoC deployment includes a single SDN controller, an application layer which implements specific functions that are intended to operate over the network via NBI and a wireless network layer. The wireless network layer is composed of pairs of devices and mediators and interoperates with the SDN controller via NETCONF/YANG interface. Five use cases were implemented as SDN applications and tested successfully demonstrating the viability of the SDN concept for wireless transport on configuring and management of wireless network elements, focused on topology planning and discovery, dynamic network view, configuration, discrepancy monitoring and detection, and event handing.

\section{MULTI-TENANCY}

The 5G-Crosshaul architecture is designed in such a way that multiple providers can share a common transport infrastructure. We refer to this case as Multi-MANO. The Multi-MANO concept requires an XCI recursion to support multiple instances of the 5G-Crosshaul MANO operating on top of the set of services provided by the $\mathrm{XCI}$ instance later. The aim of the multi-tenancy features embedded on the 5G-Crosshaul architecture is to significantly reduce the CAPEX and OPEX by efficiently sharing the infrastructures maximizing their use.

A tenant is an administrative entity that owns one or more network services offered through the XCI MANO interface. In the 5G-Crosshaul domain, three kinds of tenant are distinguished:

- Over-the-Top (OTT) Service Provider: service providers which use the 5G-Crosshaul transport infrastructure to connect distributed service points. They operate in an agnostic way over the infrastructure, and they do not require control. This is the case of CDN and TV broadcasting. Between the three type of tenant in the 5G-Crosshaul architecture, the OTI requires the mildest control over the infrastructure. To meet the QoS requirements of the OTT tenant, it is enough to provision compute and storage resources and routes allocated over the appropriate technology. In Figure 4, the tenants \#3 and \#4 represents OTT service providers.

- Mobile Virtual Network Operator (MVNO): a provider of a virtual infrastructure made over a 5G-Crosshaul network. They use the 5G-Crosshaul infrastructure as a service, without owning the physical resources and with a limited control over the virtual resources assigned. The MVNO needs some

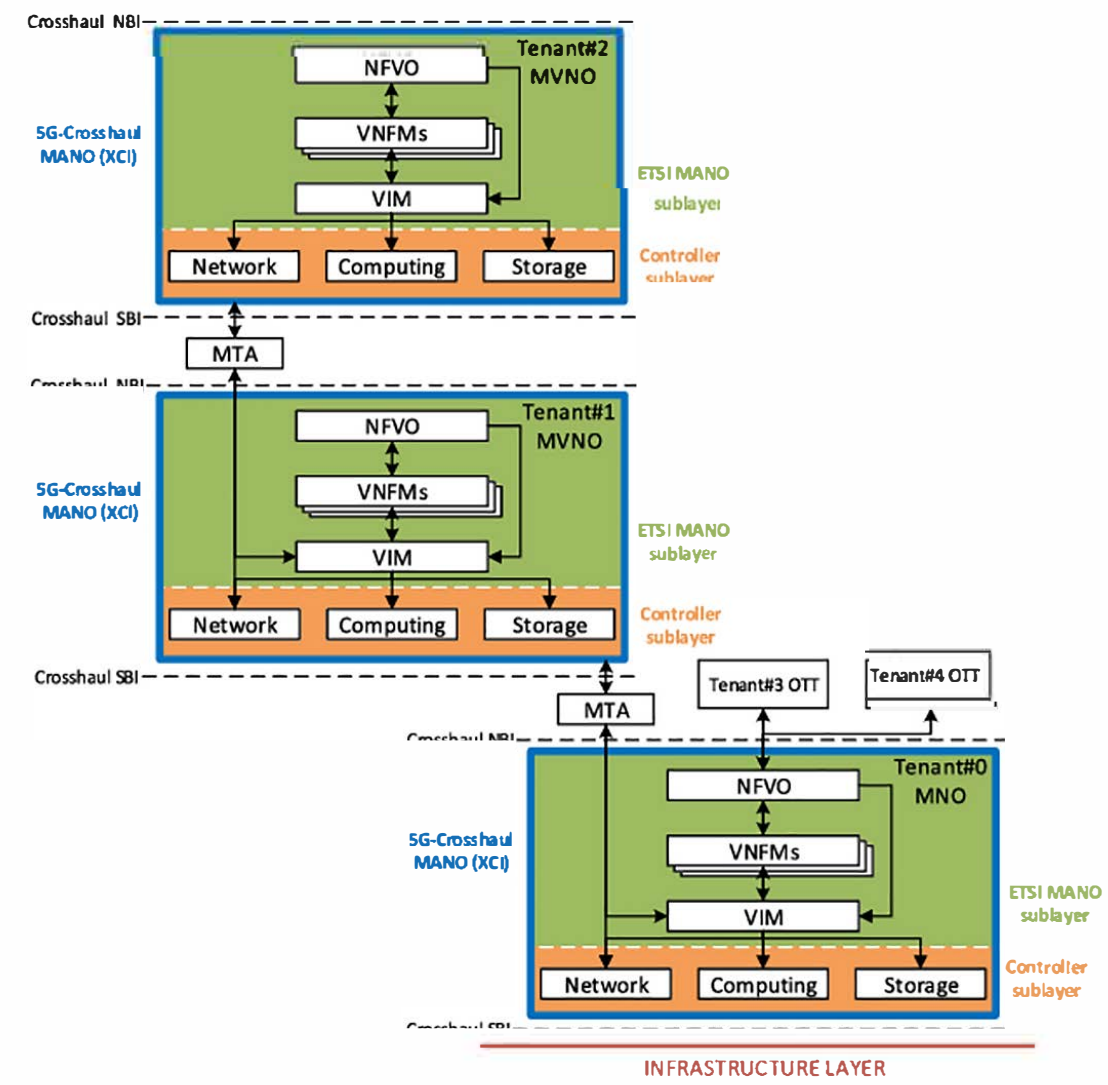

Figure 4. 5G-Crosshaul $\mathrm{XCl}$ recursive architecture. 
control over the virtual resources to manage and optimize them in base of their QoS and volume of traffic. They are denoted as Tenant\#1 and Tenant\#2 in Figure 4.

- Mobile Network Operator (MNO): it is the owner of the physical infrastructure which can serve to end users, as well as OTT or MVNO. It is the proprietor of the physical resources, so some optimization and consistency will be needed to ensure the required requests of resources by the OTT and MVNO tenants. In Figure 4 is denoted as Tenant\#0.

Figure 4 shows the 5G-Crosshaul layered recursive architecture. In the lower layer, the owner of the physical resources (MNO), instantiates its XCI, on top of the $\mathrm{MNO}$ different tenants request virtual infrastructures composed of a network subset with virtual nodes and links (i.e., a slice) to the Multi-tenancy Application (MTA), which orchestrates the assignment of the available resources. The MTA requests to the VIM the creation of a virtual topology aligned with the tenant's demand and it will exposed through the XCI NBI.

Each tenants signs a Service Level Agreement (SLA) with the MNO, now the provider must take care of meet the requirements managing the available resources. The management on top of the virtual infrastructure is performed trough an API offered by the MTA with some defined limited operations and policies.

In a recursive and hierarchical manner each tenant can operate its virtual infrastructure as the MNO operates on the physical, allocating and reselling part of the resources to other MVNOs in a hidden way to the MNO. Following this line, Figure 4 shows this practice between Tenant\#1 and Tenant\#2, the infrastructure of MVNO \#2 operates over the virtual network offered by the MVNO \#1 that operates on top of the MNO infrastructure (the physical one). In case of deploying, an OTT tenant over an infrastructure, the MTA is required to provide the tenant identification, while the mapping of the virtual to physical resource will be performed by the VIMaP (an extension of the VIM in Figure 4) through the NFVO, Figure 4 shows this practice in Tenant\#3 and Tenant\#4.

The multi-tenant architecture presented in this section is very challenging and one of the central points of innovation of the project. In order to devise a feasible and flexible framework we have followed the recursion principles of the ONF architecture [19]

This architecture also supports deployment of the network services of the OTTs on top, by extending the functions of the NFVOs such as Open Source Mano ${ }^{+*}$ and OpenBaton ${ }^{\S \S}$ to have the tenant separation and identify the mapping of a tenant to a network service consisting of a set of VNFs connected in a forwarding graph.

\footnotetext{
https://osm.etsi.org/.

$\S \S$ http://openbaton.github.io/.
}

\section{CONCLUSION}

In this paper we provide a concise view of the 5GCrosshaul architecture, introducing the main objective of transporting the backhaul and fronthaul traffic in a unified packet-based transport network. We achieve this unification with a common frame format (XCF) based on MAC-in-MAC Ethernet. Furthermore, we present the 5GCrosshaul control plane architecture, which is based on the SDN and NFV paradigms with a clear split between the logically centralized control plane (XCI) and the data plane (XFE).

To finalize the architecture discussion, we present how the multi-tenancy concept is included on it and the different types of tenant of the 5G-Crosshaul domain; moreover, the multiple recursive instantiations of the XCI allows the reselling of virtual and physical network resources over a unique physical infrastructure.

\section{ACKNOWLEDGEMENT}

This work has been funded by the EU H2020 project "5GCrosshaul: The 5G Integrated fronthaul/backhaul" (grant no. 671598).

\section{REFERENCES}

1. Cisco White Paper. Cisco Visual Networking Index: Forecast and Methodology, 2013-2018, June 2014. Available from: http://www.cisco.com/c/en/us/solutions/ collateral/service-provider/visual-networking-index-vni/ complete-white-paper-c11-481360.html [accessed on 28 June 2016].

2. Open Networking Foundation (ONF). SDN Architecture, Issue 1.1, 2016. Available from: https:// www.opennetworking.org/images/stories/downloads/ sdn-resources/technical-reports/TR-521_SDN_ Architecture_issue_1.1.pdf [accessed on 28 June 2016].

3. de la Oliva A, Hernández JA, Larrabeiti D, Azcorra A. An overview of the CPRI specification and its application to C-RAN-based LTE scenarios. IEEE Communications Magazine 2016; 54(2): 152-159.

4. IMT-2020 (5G) Promotion Group. 5G Network Technology Architecture White Paper. Available from: www. imt-2020.cn/en/documents/download/64 [accessed on 28 June 2016].

5. Next Generation Mobile Networks (NGMN) Alliance. $5 G$ White Paper. Available from: https://www.ngmn. org/uploads/media/NGMN_5G_White_Paper_V1_0.pdf [accessed on 28 June 2016].

6. Agyapong P, Iwamura M, Staehle D, Kiess W, Benjebbour A. Design considerations for a $5 \mathrm{G}$ network architecture. IEEE Communications Magazine 2014; 52 (11): 65-75. 
7. Chanclou P. Technical options for NGPON2 beyond $10 G$ $P O N$, 2011. ECOC Technical Digest.

8. Dehos C, González JL, De Domenico A, Kténas D, Dussopt L. Millimeter-wave access and backhauling: the solution to the exponential data traffic increase in $5 \mathrm{~g}$ mobile communications systems? IEEE Communications Magazine 2014; 52(9): 88-95.

9. Sabella R, Bianchi A, Bottari G, Cavaliere F, Iovanna P, Testa F. Trends in optical transport networks and related technologies. In 16th International Conference on Transparent Optical Networks (ICTON), Graz, Austria, 2014.

10. Jungnickel V, Vucic J, Langer K-D. High-speed optical wireless communications. In Optical Fibre Conference (OFC), San Francisco, CA, USA, 2014.

11. Fabrega J M, Svaluto Moreolo M, Jimenez F, Chochol M, Junyent G. Constant envelope coherent optical OFDM transceiver for elastic upgrade of transport network. In 16th International Conference on Transparent Optical Networks (ICTON), Graz, Austria, 2014.

12. Open Base Station Architecture Initiative (OBSAI). OBSAI System Spec V2.0. Available from: http:// www.obsai.com/specs/OBSAI_System_Spec_V2.0.pdf [accessed on 28 June 2016].

13. IEEE 1904 Task Group. Standard for Radio Over Ethernet Encapsulations and Mappings.
14. Chih-Lin I, Yuan Y, Huang J, Ma S, Cui C, Duan R. Rethink fronthaul for soft RAN. IEEE Communications Magazine September 2015; 53(9): 82-88.

15. IEEE 802.1 Task Group. IEEE 802.1 - Time-Sensitive Networking.

16. ETSI, Network Functions Virtualisation. Network Functions Virtualisation (NFV); Management and Orchestration, December 2014. Available from: http:// www.etsi.org/deliver/etsi_gs/NFV-MAN/001_099/001/ 01.01.01_60/gs_nfv-man001v010101p.pdf [accessed on 28 June 2016].

17. IEEE 802.1 Task Group. IEEE 802.1ah-2008 - IEEE Standard for Local and Metropolitan Area Networks Virtual Bridged Local Area Networks Amendment 7: Provider Backbone Bridges.

18. Open Networking Foundation (ONF). OpenFlow Switch Specification 1.5.1. Available from: https://www.open networking.org/images/stories/downloads/sdn-resources/ onf-specifications/openflow/openflow-switch-v1.5.1.pdf [accessed on 28 June 2016].

19. Open Networking Foundation (ONF). SDN Architecture. Available from: https://www.opennetworking.org/images/ stories/downloads/sdn-resources/technical-reports/TR SDN_ARCH_1.0_06062014.pdf [accessed on 28 June 2016]. 